$\xi=$

\title{
Emergency call receiving alert through mobile phones using to change the current profile
}

\author{
Siva Rama Lingham $\mathrm{N}^{1}$, G. Yuvaraj ${ }^{1}$, T. Senthil Murugan ${ }^{1}$ \\ ${ }^{I}$ Assistant Professor, Department of Computer Science and Engineering, School of Computing, Vel Tech Rangarajan Dr. Sagunthala \\ $R \& D$ Institute of Science and Technology, Avadi, Chennai - 600 062, Tamil Nadu, India \\ *Corresponding author E-mail: sivaramalinghamn@veltech.edu.in
}

\begin{abstract}
Emergency Call Alert (ECA) Application is developed for inform the urgency as well as seriousness of the call to the receiver. The profile in the mobile facilitates that the user to change the mode they need and this requires manual work load. This Call Manager changes the pro-file automatically to the desired mode designed by the user. It has also been enhanced with filtering mechanism called as Emergency calls filter which allows the calls from the emergency list to be in user preferred mode irrespective of the current mode. This feature is to avoid the missing of calls from the numbers specified in the emergency call list. Now a day most of calls come to us when we are busy, it is an interrupt, which disturbs or sometimes accident may happen. To avoid this either we switch off the mobile or we put the mobile in silent mode. If this is so if any emergency call comes we cannot attend such type of calls. The other disadvantage of this is the calling person does not know when the called person is free. To overcome all these problems we presented paper, Emergency Call Manager which handles all the call when he/she is busy sends an SMS. Software which filters this message and it gives an emergency alarm to called mobile.
\end{abstract}

Keywords: Emergency Call Manager; Call Filter; Message Service.

\section{Introduction}

Though mobile phone provides a wide variety of features, it sometimes acts as a hindrance during working hours. This project is mainly designed for averting the disturbance caused by the mobile phone to the staff members during class hours. When the staff members take class, an incoming call may be felt as a disturbance to both the concerned staff and students. To avoid this disturbance, the current profile system in mobile phones provides a provision of activating the silent mode. Nevertheless, the difficulty faced by this system is that, for every class hour the staff has to switch his/her mobile to silent mode manually. It increases the manual work. Hence, to avoid this problem this project has been designed. The objective of Call manager is to overcome the inconvenience experienced during working hours due to the rudiment profile activation system in mobile phones.

Call Manager switches the profile automatically to the desired mode based on the input from the user. It has also been enhanced with filtering mechanism called as Emergency calls filter, which allows the calls from the emergency list to be in General mode irrespective of the user mode. This feature is to avoid the missing of calls from the numbers, which the user feels as important. The other feature of this call manager is to send a message to the call originator for the unanswered call to know that the receiver is busy at work. This avoids the unnecessary irritation faced by the call originator about the unanswered call. The security feature is also provided in the call manager to deny the access to unauthorized users.

\subsection{Objective}

Now a day is the mobile users like advertisement, collecting funds, unwanted bank calls and so on, receive so many calls. Due to avoiding such calls, the mobile users are changing the profiles for maintaining silence and they are not considered the emergency calls. Hence, overcome these issues, the proposed application will collect the emergency phone numbers from the users. Normally, In case of emergency (ICE) has activated. Nevertheless, in this method, the receiver receives the phone call then the system will check emergency numbers database. If a number is there, it will change the profile automatically from silent mode to loud mode [1]. Even the receiver not able to attend, the system will send the message like user may busy try after sometime to dialer.

\section{Related work}

The proposed system is designed to attend emergency call using android app. A provision to avoid the missing of emergency calls irrespective of the profile mode is provided. An intimation system, which sends messages to the call originator denoting the receiver's Inability to answer the call.

The some of the main advantage over here is to avoid the missing of emergency calls irrespective of the user profile is provided in the application. An intimation system, which sends messages to the call originator denoting the receiver's inability to answer the call, is enabled.

The MobileLoud Android application has been used with the help of emergency call intimation and call originating. And one more additional added value here is to access our mobile devices any- 
where in the region is to process the call maintenance with the help of request and processing of HTTP [2]. This Mobile Loud is used to process some API's determinations as followed in the Emergency call manager.

\section{Block diagram}

This architecture shows how the process flows carry on in the Emergency call manager. First in the instance, it will create the database with the help of Emergency numbers and it will be added in the phonebook database [3]. So here we not need any GPS tracking and all. Mode conversation take place is to take a conversion between the users after connecting the call. If the call is not connected with the emergency number first time this will generate a message to the respective caller says that 'the user is busy right now' you can make the call later. User can add the more number of emergency numbers into the respective database of their individual phonebook. An authorize User can able to change their profile settings by their own like loud or mute or vibrate mode. So this is a user defined setting, hence switching is to be done by user only.

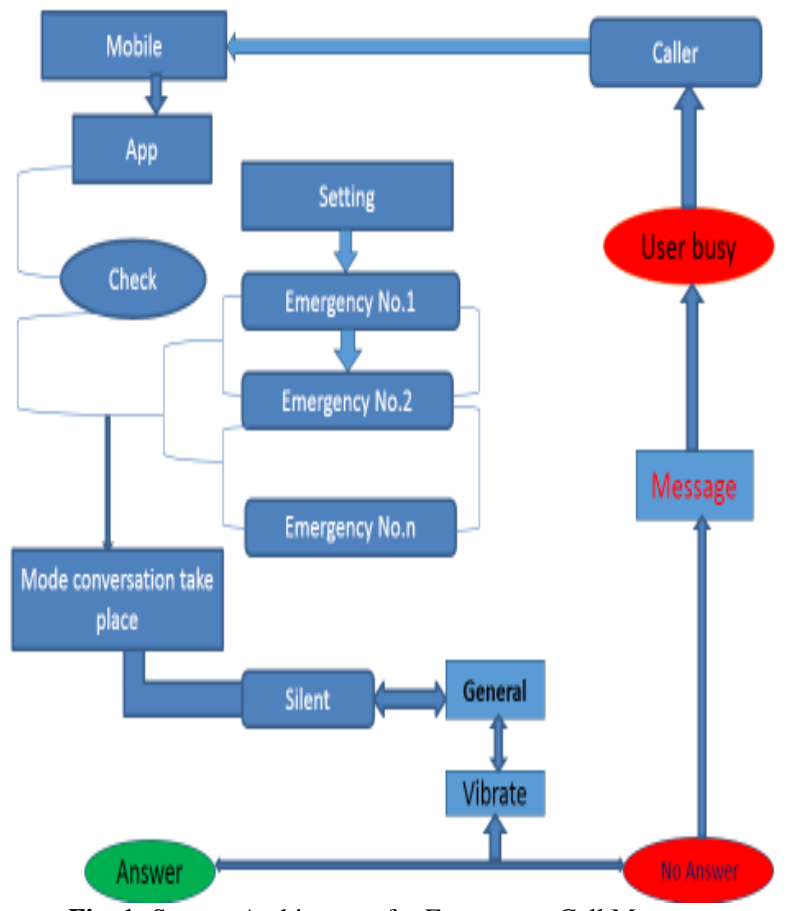

Fig. 1: System Architecture for Emergency Call Manager.

\section{Working principle}

Normally we have add the phone numbers in our phone book Then the particular user or caller has to modify his own settings on their own mobile devices. On getting incoming call, the profile will be auto switched to loud by using mobileloud app. In addition, is to generate the automatic message reply to the call originator who made the call for the inconsistent situation. Here Both the call originator and call receiver belonging to each other. It is fully based on authorized security access of auto profile management in their individual mobile devices.

The Software is divided into separately named and addressable components called modules that are integrated to satisfy problem requirements. Modularity is the single attribute of software that allows a program to be intellectually manageable.

An Emergency Call Manager in Silent Mode Using Android App application is divided into five different modules. They are

1) Creating Application (Mobile Loud) - This allows user to create and application according to their perspective scenario.

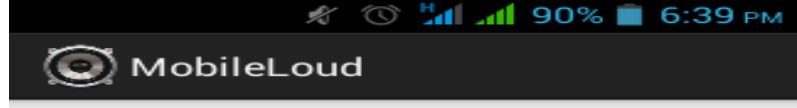

Silent to Loud

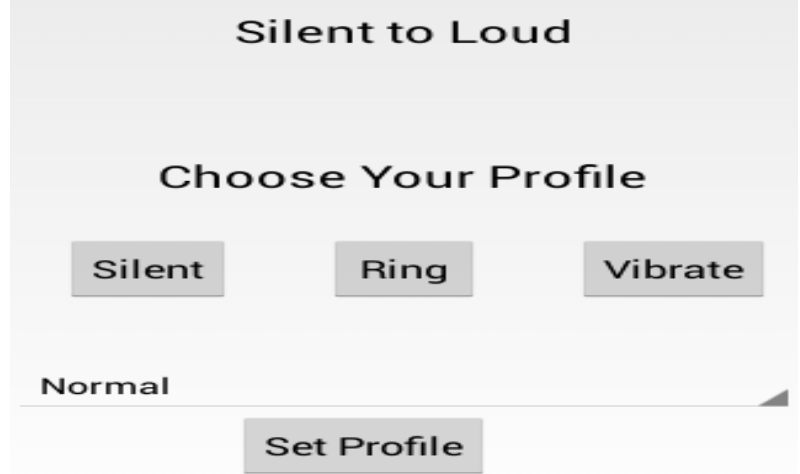

Fig. 2: Example of an Image for Mobile in Silent Mode.

2) Broadcast Receiver - Broad cast receiver simply responds to broadcast messages from other application or from the system itself. These messages are sometime called events or intents.

For example, applications can also initiate broadcasts to let other applications know that some data has been downloaded to the device and is available for them to use, so this is broadcast receiver who will intercept this communication and will initiate appropriate action. A broadcast receiver is implemented as a subclass of Broadcast Receiver class and each message is broadcasted as an Intent object.

3) Sound Manager - Sound manager exposes android's sound $\&$ volume controls. It provides a quick way to change ring mode or vibration settings, or mute and unmute your phone.

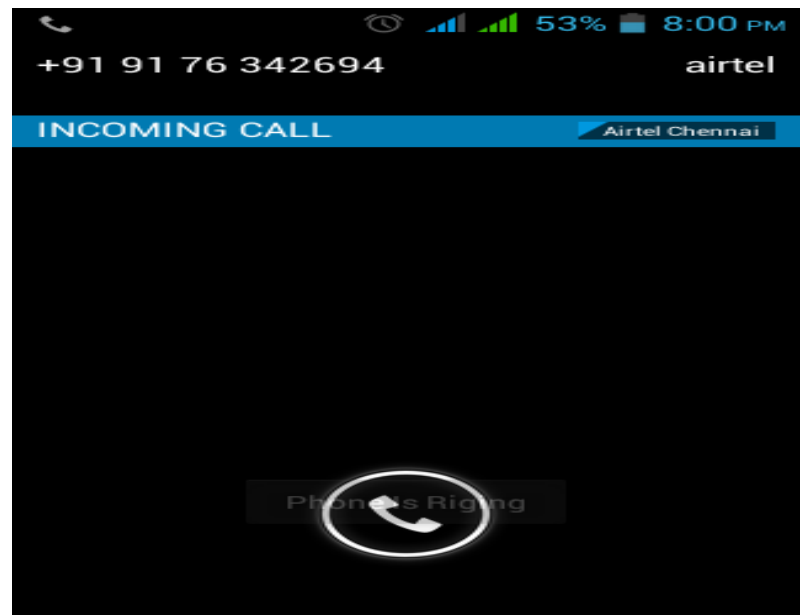

Fig. 3: Example of an Image for Switching the Profile.

4) Call Filter - Emergency calls filter allows the calls from the emergency list to be in General or vibrate mode irrespective of the current mode of the profile. 


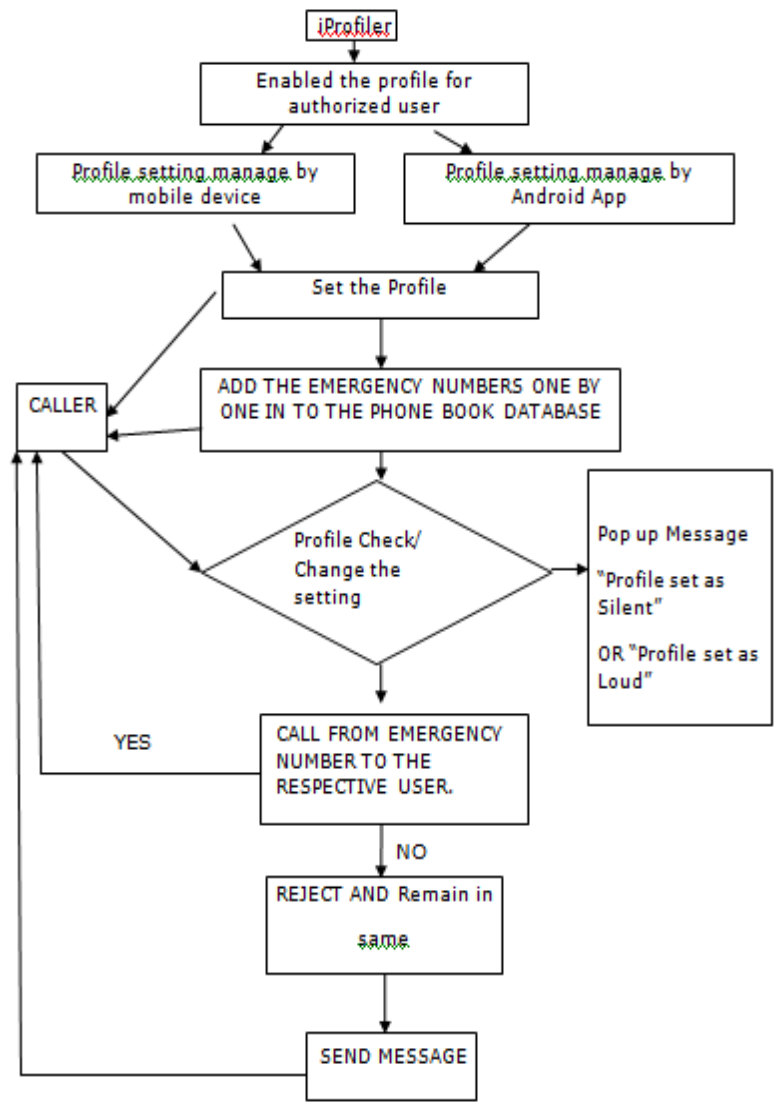

Fig. 4: Process Flow in Profile Changing System.

5) SMS Services - Allows the user to send a message to the call originator for the unanswered call informing that the receiver is busy at work.

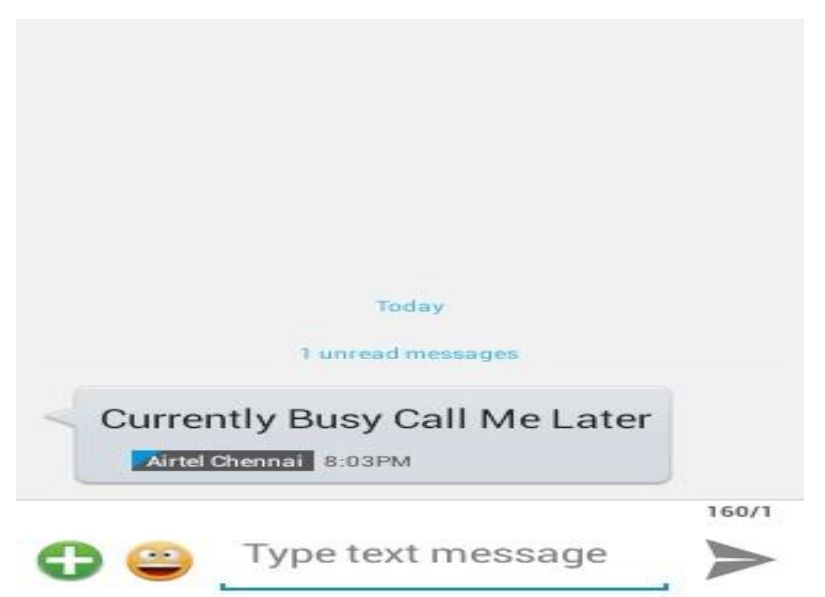

Fig. 5: Example Image for Message to the Call Originator.

Based on the above modules the user authentication and process carried for the automatic switching system of mobile profile termed as iProfiler.

\section{Conclusion}

Mobile communication uses more and more expertise in their controls without using wired networks. In this paper, an effective way of passing information to the respective user at the time that is our main goal with the help of mobileloud application. This deals with an automated tool for Level Crossing Bridge over mobile communications. It will mainly reduce the intervention of every human beings and using mobileloud app in android is make ourself to become more familiar and smarter with the android as well as everyone can handle the situation of emergency also an smarter and easier. In future the Mobile Loud will be enhanced for all the people who think mobile phone as disturbance in working hours. In Future the Mobile Loud application is to be enhanced for different kinds of platforms. The current system deals with only the emergency call enabling mechanism and SMS mechanism. It will be enhanced with all the network implementations of future. Mobile Loud is designed in such a way that it could be enhanced for future easily.

\section{References}

[1] A Sulochandevi, Siddhesh rane, Lalji devda, Raj Phadke, "Sound Profile Switching", IRJET International Research Journal of Engineering and Technology, Volume: 04 Issue: 03, March 2017.

[2] Manav Singhal, Anupam Shukla, "Implementation of Location based Services in Android using GPS and Web Services", IJCSI International Journal of Computer Science, Vol. 9, Issue 1, No.2, January 2012.

[3] Mr. Magesh Kumar .S, Mr.Raj, "Emergency Application For Women", International Journal of Scientific and Research Publications, Volume 4, Issue 3, March 2014.

[4] Dr. Shantakumar B. Patil, Dr. Premjyoti Patil,Gopinath A R , "Safety with Mobile Technologies:Handle All Calls Without Disturbing The User", Proceedings of International Conference on Networking and Computer Application July 15-16, 2015.

[5] Rohit Madhukar Chaskar, "Location based automatic sound profile switching application in android mobiles", International Journal of Computer \& Communication Technology, Volume-4, Issue 2, 2013.

[6] Rajdip kaur Bath, Tyson Fernandes, Akash Jadhav, "Profile Management System", IJCSIT - International Journal of Computer Science and Information Technology, Vol. 6 (2), 2015.

[7] Kore Geetanjali T, Naik Suman R, Sanap Kavita V, Mawale Ashwini J, "Autosilent Mobile System", International Journal of Advanced Research in Computer Engineering \& Technology, Volume 3 Issue 3, March 2014 